\title{
Glycolysis-associated enzymes existing in the follicular lumen of the thyroid may interfere with energy metabolism
}

\author{
HUIBIN HUANG ${ }^{1}$, YAXIONG SHI ${ }^{1}$, HUIYAO CAI $^{1}$, BO LIANG $^{1}$, HONGHONG DUAN $^{2}$ and QINGYAN CAI $^{1}$ \\ Departments of ${ }^{1}$ Endocrinology and ${ }^{2}$ Gynecology and Obstetrics, The Second Affiliated Hospital \\ of Fujian Medical University, Quanzhou, Fujian 362000, P.R. China
}

Received May 10, 2016; Accepted July 11, 2016

DOI: $10.3892 /$ br.2016.721

\begin{abstract}
Synthesis and storage of the thyroid hormone precursor, thyroglobulin (TG), occurs within the follicular lumen of the thyroid and the TG is then absorbed into cells for further processing before release into the blood. However, the mechanism of energy metabolism in the follicular lumen of the thyroid remains unknown. In the present study, the three dimensional structure of thyroid follicles was constructed using a primary culture of swine cells and the follicular protein was identified via matrix-assisted laser desorption/ionization-time of flight mass spectrometry. Three glycolysis-associated enzymes, enolase, pyruvate kinase and phosphoglyceraldehyde dehydrogenase were identified in addition to TG. These results support the hypothesis that anaerobic glycolysis of glucose exists in the follicle and supports energy consumption for hormone synthesis.
\end{abstract}

\section{Introduction}

The thyroid follicle, which is the fundamental unit of the thyroid, consists of polarized thyroid cells. The synthesis and secretion of thyroid hormone depends on the integrity of the thyroid follicular structure, as well as the uptake of iodine $(1,2)$. The follicular lumen is an enclosed cavity, where the intermediate processes of thyroid hormone synthesis, such as iodine activation and iodination of thyroglobulin (TG) take place. However, under anaerobic conditions, synthesis is completed outside the cells. Currently, the energy source supporting follicular hormone synthesis is unknown.

In the present study, the three dimensional structure of the follicle was constructed in vitro using a primary culture of swine thyroid cells and the protein was extracted from the follicular lumen (3). Proteomics analysis was subsequently

Correspondence to: Dr Huibin Huang, Department of Endocrinology, The Second Affiliated Hospital of Fujian Medical University, 34 North Zhongshan Road, Quanzhou, Fujian 362000, P.R. China

E-mail: huibinhuang@aliyun.com

Key words: thyroid, glycolysis, follicle, enzyme conducted to identify the unknown follicular lumen proteins that were associated with energy metabolism. The aim of the study was to improve the understanding of the mechanism of energy metabolism in the follicular lumen.

\section{Materials and methods}

Preparation of the thyroid monolayer and three-dimensional cell culture. Adult swine were maintained in Qingyuan Farm (Quanzhou, China). The present study was approved by the ethics committee of the Second Affiliated Hospital of Fujian Medical University (Fujian, China). Two adult swine were sacrificed by carotid artery bleeding and the thyroid gland was removed immediately. Subsequent to serial washing with milli-Q water, 75\% ethanol, and sterilized phosphate-buffered saline (PBS; Thermo Fisher Scientific, Inc., Shanghai, China), the capsule and overlaying tissues were peeled away. The remaining thyroid tissue was minced into $1-\mathrm{mm}^{3}$ sections. Following digestion with $0.125 \%$ trypsin (Thermo Fisher Scientific, Inc.) for $30 \mathrm{~min}$ at room temperature and filtering through a mesh sieve (size, 200; BD Biosciences, Shanghai, China), the cell suspension was inoculated in 6-well plates, and maintained in a $37^{\circ} \mathrm{C}$ incubator $\left(5 \% \mathrm{CO}_{2}\right)$ in Dulbecco's modified Eagle's medium and Ham's F-12 medium (Thermo Fisher Scientific, Inc.) consisting of $1 \mathrm{mU} / \mathrm{ml}$ thyroid-stimulating hormone, $0.05 \% \mathrm{NaI}$ and $10 \%$ fetal bovine serum (Thermo Fisher Scientific, Inc.). For construction of the three-dimensional thyroid follicle, the cells were seeded at a density of $2 \times 10^{6}$ cells $/ \mathrm{ml}$, and the thyroid follicle was formed three days after inoculation (Fig. 1A-D).

Extraction of thyroid follicular lumen and intracellular proteins. The follicular lumen protein was prepared as follows: After aspiration of the culture medium from the culture plate, the three-dimensional thyroid cells were gently washed, twice, with pre-cooled PBS, followed by incubation with $0.02 \%$ EDTA for $5 \mathrm{~min}$ at room temperature. EDTA loosened the thyroid structure. After removing the EDTA solution, the thyroid structure was mechanically deconstructed using $100 \mu \mathrm{l}$ PBS and the protein was released from the follicular lumen. The follicular lumen protein concentration was diluted to a similar concentration to thyroid intracellular protein using bovine serum albumin (BSA). For preparing the intracellular protein, the solution was centrifuged at $12,000 \mathrm{x} \mathrm{g}$ for $5 \mathrm{~min}$ 

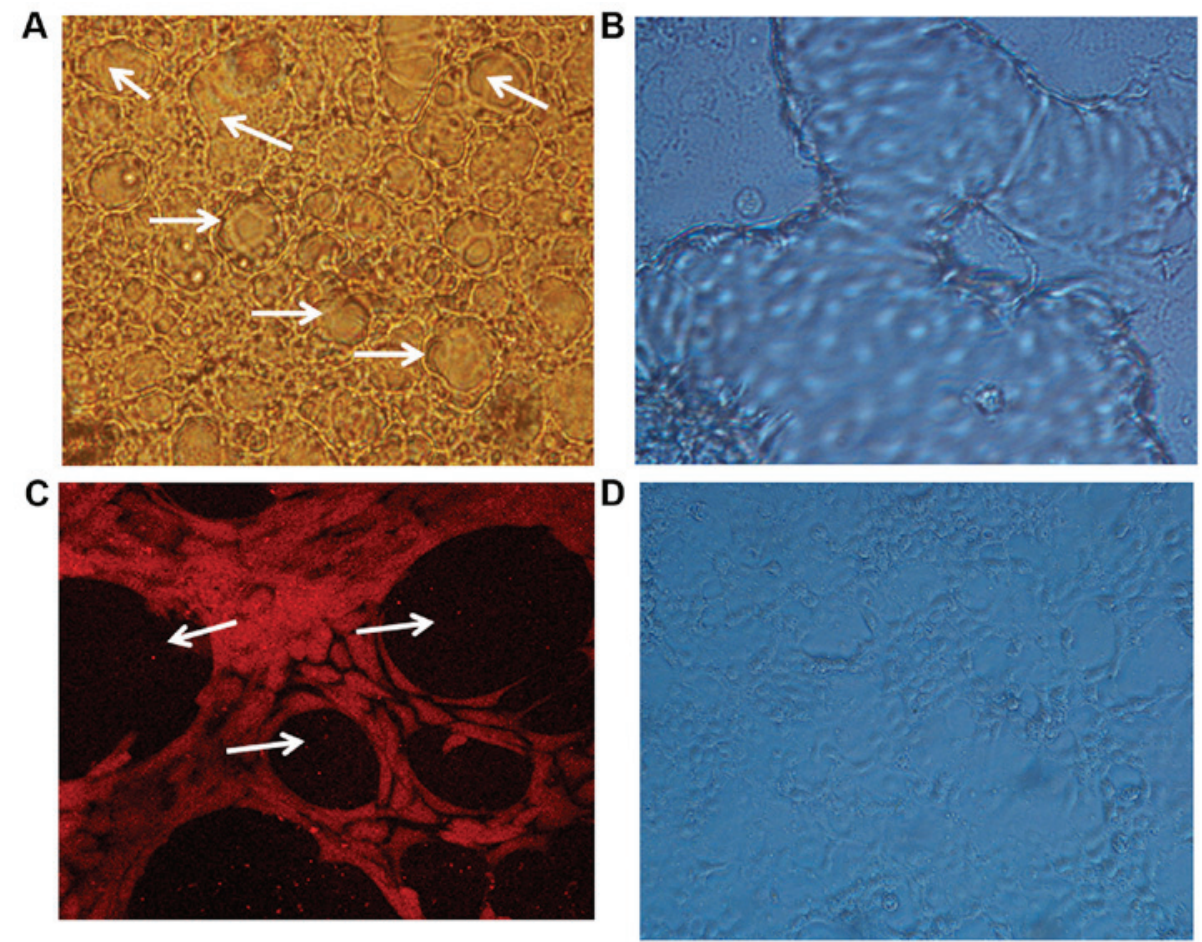

Figure 1. Morphological observation of reconstituted thyroid follicles. (A-C) Reconstituted thyroid follicles were elliptical and round. Images were obtained by phase contrast microscopy. (C) The follicular lumen of the round-shaped reconstituted follicles. (D) A laser confocal microscope was employed to scan the follicles following staining with anti-thyroglobulin. The arrows indicate thyroid follicles. Magnification: A, x100; B and C, x200; D, x50.

at $4^{\circ} \mathrm{C}$ and the supernatant was aliquoted. The cell pellet was lysed using RIPA buffer (Santa Cruz Biotechnology, Inc., Dallas, TX, USA) and centrifuged at $12,000 \mathrm{x}$ for $10 \mathrm{~min}$ at $4^{\circ} \mathrm{C}$ to harvest the intracellular protein. The protein concentration was determined using a Micro BCA Protein Assay kit (Thermo Fisher Scientific, Inc.) according to the manufacturer's instructions.

SDS-PAGE electrophoresis and matrix assisted laser desorption/ionization time-of-flight mass spectrometry (MALDI-TOF) analysis. For SDS-PAGE electrophoresis, $20 \mu \mathrm{g}$ protein was loaded and separated on 4 or $12 \%$ polyacrylamide gels according to the standard protocol. After running for $1 \mathrm{~h}$ at $120 \mathrm{~V}$, the gel was stained with Coomassie Brilliant Blue. The protein bands that were differentially expressed and highly overexpressed in the follicular lumen were sliced from the gel and digested using $12.5 \mathrm{ng} / \mu \mathrm{l}$ trypsin in $50 \mathrm{mmol} / \mathrm{l}$ ammonium bicarbonate ( $\mathrm{pH}$ 8.0). Following digestion, the samples were eluted with $2 \mu \mathrm{l}$ matrix solution containing $10 \mathrm{mg} / \mathrm{m} \alpha$ cyano-4-hydroxycinnamic acid, and submitted to Bruker III MALDI-TOF mass spectrometry. The trypsin autolysis products were used for calibration by flexAnalysis software (version 2.4; Bruker, Coventry, UK) and searched against the SWISS-PROT (http://www.uniprot.org/) and NCBI database (http://www.ncbi.nlm.nih.gov/) using the MASCOT tool from Matrix Science (http://www.matrixscience.com/) with a $50 \mathrm{ppm}$ mass tolerance.

Imaging of monolayer thyroid cells and follicular lumen. Primary thyroid cells were isolated as described above. Then $6 \times 10^{6}$ cells per well were inoculated into 6 -well plates for reconstructing the three dimensional structure and $6 \times 10^{5}$ cells per well for monolayer cells. After continuous cultivation, cells growing in a monolayer fashion were visualized and photographed under a normal inverted optical microscope. For laser confocal microscopy, the cells forming the follicular lumen were gently rinsed with PBS twice followed by incubation with fluorophore-conjugated anti-TG (Novus Biologicals LLC, Littleton, CO, USA) for $1 \mathrm{~h}$ at room temperature. After three washes with PBS, the cells were visualized under a laser confocal microscope.

Statistical analysis. Statistical significance was determined using the one-way analysis of variance (SPSS 18.0; SPSS, Inc., Chicago, IL, USA). The results were expressed as means \pm standard error of the mean and $\mathrm{P}<0.05$ was considered to indicate a statistically significant difference.

\section{Results}

Gel analysis of the thyroid intracellular and follicular lumen proteins. In order to visualize the abundance of proteins, the intracellular protein and follicular lumen proteins were separated by SDS-PAGE (4 and 12\%) according to standard procedures, using the total proteins that were extracted from the thyroid epithelial cells with RIPA buffer. Equal quantities of total protein were loaded into each lane. After staining with Coomassie Brilliant Blue, certain follicular lumen proteins were observed to be differentially expressed compared with the control intracellular proteins. These are demonstrated in Fig. 2A and B.

Identification of differentially expressed proteins by mass spectrometry. Five protein bands were excised and digested 


\section{A}
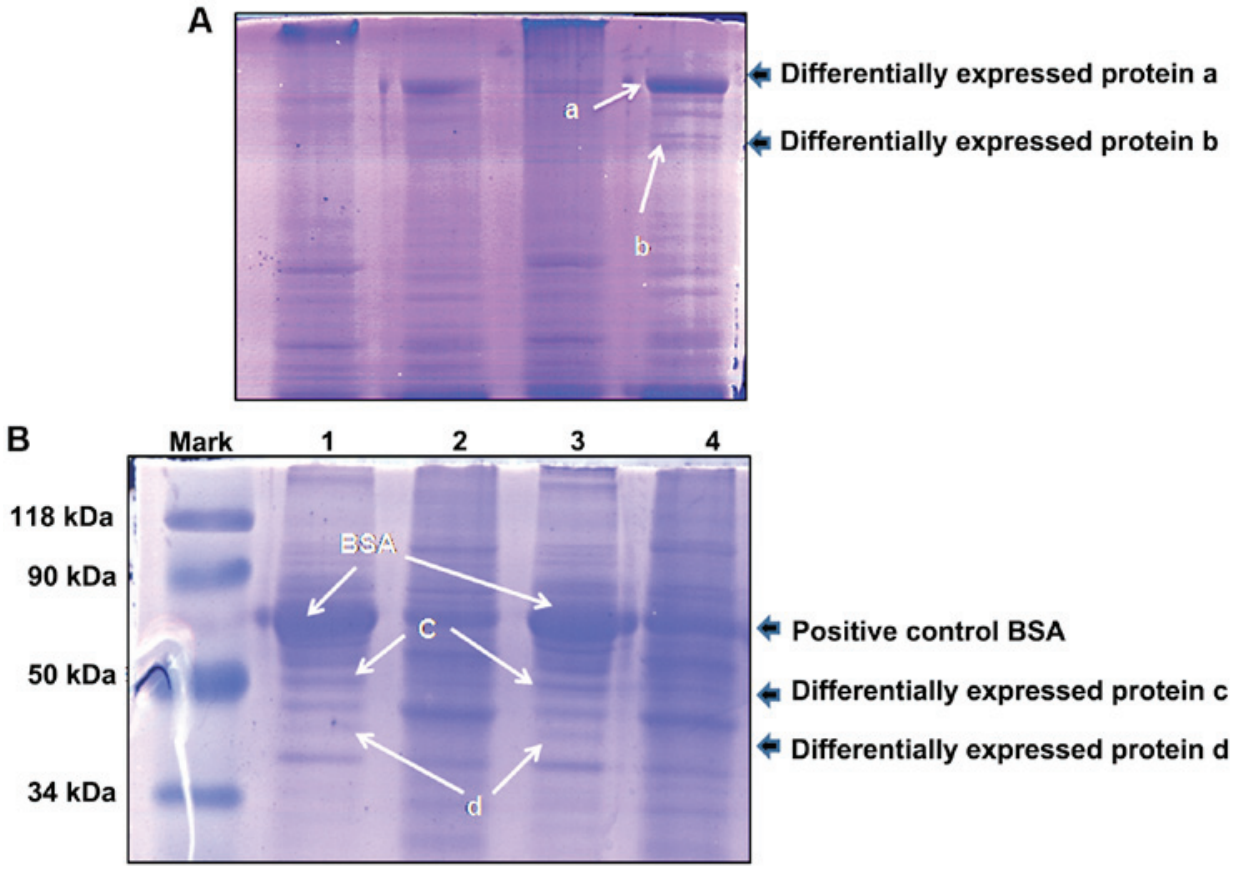

Figure 2. (A) 4\% SDS-PAGE electrophoresis of thyroid intracellular and follicular lumen proteins. Lanes 1 and 3, intracellular proteins; lanes 2 and 4 , follicular lumen proteins. The arrow indicates the differentially expressed proteins. (B) 12\% SDS-PAGE electrophoresis of thyroid intracellular protein and follicular lumen proteins. Lanes 1 and 3, follicular lumen proteins; lanes 2 and 4, intracellular proteins. The arrow indicates the differentially expressed proteins. Protein a, thyroglobulin (Sus scrofa); protein b, enolase; protein c, pyruvate kinase; protein d, phosphoglyceraldehyde dehydrogenase. BSA, bovine serum albumin.

Table I. Differentially expressed follicular proteins as identified by mass spectrometry.

\begin{tabular}{lllrr}
\hline Band & \multicolumn{1}{c}{ Protein } & Accession no. & Molar mass calculation (Da) & Score \\
\hline Protein a & Thyroglobulin (Sus scrofa) & gil270289746 & 299851 & 302 \\
Protein b & Enolase & gil34789 & 47285 & 111 \\
Protein c & Pyruvate kinase & gil206205 & 58314 & 292 \\
Protein d & Phosphoglyceraldehyde dehydrogenase & gil65987 & 35914 & 172 \\
\hline
\end{tabular}

with trypsin, then identified by mass spectrometry. The identified proteins, TG, enolase, pyruvate kinase and phosphoglyceraldehyde dehydrogenase are presented in Table I.

\section{Discussion}

The thyroid follicle is the fundamental unit of the thyroid gland with round or elliptical shaped cells that are 20-900 $\mu \mathrm{m}$ in diameter. Monolayer follicular endothelial cells aggregate to form a lumen, which is filled with TG $(2,4)$. TG is considered to be the carrier for thyroid hormone synthesis. The biosynthesis of thyroid hormone is a process of chemical modification of TG $(5,6)$. Follicular endothelial cells transport TG and iodine into the follicular lumen and partial tyrosine in TG is catalyzed to 3-monoiodotyrosine (MIT) and 3,5-diiodotyrosine (DIT) in the apical membrane surface in the lumen by thyroid peroxidase. MIT or DIT further couple with DIT to create triiodothyronine (T3) and thyroxine (T4). TG resides in the lumen carrying the newly synthesized $\mathrm{T} 3$ and $\mathrm{T} 4$, which must be trafficked back to thyroid follicular epithelial cells for further processing prior to the release of $\mathrm{T} 3$ and $\mathrm{T} 4$ (7). The process includes the uptake of TG, degradation of iodinated TG and release of thyroid hormones. Therefore, the physiological features of thyroid follicular epithelial cells are that the synthesis and storage of thyroid hormones are conducted within the follicular lumen and are absorbed into cells for further processing before being released into the blood (8-10), which is distinct from other endocrine cells that preserve the hormone intracellularly. Thus, the follicular lumen is where thyroid hormone synthesis is performed. All these steps, including TG synthesis and transportation, and iodine uptake, consume ATP $(11,12)$; however, the mechanism by which ATP is generated in the follicular lumen remains unknown.

As an extracellular structure, the thyroid follicular lumen is isolated from the blood and cannot access oxygen support; therefore, it is proposed that the energy supply in the lumen is via anaerobic glycolysis, and that anaerobic glycolysis-associated enzymes must exist in the lumen. To analyze this hypothesis, the follicular lumen was constructed using a primary culture of swine thyroid cells in the present study. In addition, the soluble protein was extracted from the lumen and SDS-PAGE was performed to separate the follicular lumen 
proteins. It must be noted that, as the follicular lumen proteins are not abundant, each band in the gel may have represented a single protein. Furthermore, as a result of the method that was used to isolate the thyroid follicular lumen proteins, intracellular proteins may have been mixed into the samples. Equal quantities of total protein (thyroid intracellular or follicular lumen proteins) were loaded into each lane for SDS-PAGE gel electrophoresis and those protein bands that were highly overexpressed in the follicular lumen or were absent in the thyroid intracellular protein represented follicle-specific proteins. Following electrophoresis, five follicle-specific proteins were identified by MALDI-TOF mass spectrometry. The band with $60 \mathrm{kDa}$ MW was BSA, and the other bands were swine TG, enolase, pyruvate kinase and phosphoglyceraldehyde dehydrogenase. Among these five proteins, BSA served as a positive control protein that was artificially supplemented, swine TG was previously shown to be present in the follicular lumen, and the other three proteins were key glycolysis-associated enzymes, which have previously been identified to be critical in anaerobic glycolysis of glucose (13-15).

In conclusion, anaerobic glycolysis of glucose commonly occurs in the cytoplasm and is the major energy source when cells are subjected to hypoxia (16-18). The present study demonstrated that these glycolysis-associated enzymes, enolase, pyruvate kinase and phosphoglyceraldehyde dehydrogenase exist in the follicular lumen; however, their roles in the lumen remain undefined, and further investigation is required to establish whether anaerobic glycolysis of glucose also occurs in the follicular lumen and supports the energy consumption for hormone synthesis.

\section{Acknowledgements}

The present study was supported by the Natural Science Foundation of Fujian (grant no. 2012J01332), the Natural Science Foundation of China (grant no. 81370886), the Key Scientific Project of Fujian Province (grant no. 2014Y0017) and the Innovative Medical Research Project of Fujian Province (grant no. 2012-CXB-24).

\section{References}

1. Susarla R, Gonzalez AM, Watkinson JC and Eggo MC: Expression of receptors for VEGFs on normal human thyroid follicular cells and their role in follicle formation. J Cell Physiol 227: 1992-2002, 2012.

2. Bernier-Valentin F, Trouttet-Masson S, Rabilloud R, Selmi-Ruby $S$ and Rousset B: Three-dimensional organization of thyroid cells into follicle structures is a pivotal factor in the control of sodium/iodide symporter expression. Endocrinology 147: 2035-2042, 2006.

3. Huang H, Shi Y, Lin L, Li L, Lin X, Li X and Xu D: Inhibition of thyroid-restricted genes by follicular thyroglobulin involves iodinated degree. J Cell Biochem 112: 971-977, 2011.
4. Tonoli H, Flachon V, Audebet C, Callé A, Jarry-Guichard T, Statuto M, Rousset B and Munari-Silem Y: Formation of three-dimensional thyroid follicle-like structures by polarized FRT cells made communication competent by transfection and stable expression of the connexin-32 gene. Endocrinology 141: 1403-1413, 2000.

5. Di Jeso B and Arvan P: Thyroglobulin from molecular and cellular biology to clinical endocrinology. Endocr Rev 37: 2-36, 2016.

6. Ishido Y, Luo Y, Yoshihara A, Hayashi M, Yoshida A, Hisatome I and Suzuki K: Follicular thyroglobulin enhances gene expression necessary for thyroid hormone secretion. Endocr J 62: $1007-1015,2015$

7. Hennemann G, Vos RA, de Jong M, Krenning EP and Docter R: Decreased peripheral 3,5,3'-triiodothyronine (T3) production from thyroxine (T4): A syndrome of impaired thyroid hormone activation due to transport inhibition of T4- into T3-producing tissues. J Clin Endocrinol Metab 77: 1431-1435, 1993.

8. Miot F, Dupuy C, Dumont J and Rousset B: Thyroid hormone synthesis and secretion. In: Endotext. De Groot LJ, Beck-Peccoz P, Chrousos G, Dungan K, Grossman A, Hershman JM, Koch C, McLachlan R, New M, Rebar R, Singer F, Vinik A, Weickert MO (eds). MDText.com, Inc., South Dartmouth, MA, 2000.

9. Koibuchi N: Molecular mechanisms of thyroid hormone synthesis and secretion. Nihon Rinsho 70: 1844-1848, 2012 (In Japanese).

10. Vickers AE, Heale J, Sinclair JR, Morris S, Rowe JM and Fisher RL: Thyroid organotypic rat and human cultures used to investigate drug effects on thyroid function, hormone synthesis and release pathways. Toxicol Appl Pharmacol 260: 81-88, 2012.

11. Massart C, Hoste C, Virion A, Ruf J, Dumont JE and Van Sande J: Cell biology of $\mathrm{H}_{2} \mathrm{O}_{2}$ generation in the thyroid: investigation of the control of dual oxidases (DUOX) activity in intact ex vivo thyroid tissue and cell lines. Mol Cell Endocrinol 343: 32-44, 2011.

12. Corvilain B, Laurent E, Lecomte M, Vansande J and Dumont JE: Role of the cyclic adenosine 3',5'-monophosphate and the phosphatidylinositol- $\mathrm{Ca}^{2+}$ cascades in mediating the effects of thyrotropin and iodide on hormone synthesis and secretion in human thyroid slices. J Clin Endocrinol Metab 79: 152-159, 1994.

13. Li W, Xu Z, Hong J and Xu Y: Expression patterns of three regulation enzymes in glycolysis in esophageal squamous cell carcinoma: association with survival. Med Oncol 31: 118, 2014.

14. Slamovits $\mathrm{CH}$ and Keeling PJ: Pyruvate-phosphate dikinase of oxymonads and parabasalia and the evolution of pyrophosphate-dependent glycolysis in anaerobic eukaryotes. Eukaryot Cell 5: 148-154, 2006.

15. Atlante A, Giannattasio S, Bobba A, Gagliardi S, Petragallo V, Calissano P, Marra E and Passarella S: An increase in the ATP levels occurs in cerebellar granule cells en route to apoptosis in which ATP derives from both oxidative phosphorylation and anaerobic glycolysis. Biochim Biophys Acta 1708: 50-62, 2005.

16. Pietsch J, Sickmann A, Weber G, Bauer J, Egli M, Wildgruber R, Infanger $M$ and Grimm D: Metabolic enzyme diversity in different human thyroid cell lines and their sensitivity to gravitational forces. Proteomics 12: 2539-2546, 2012.

17. Zhou L, Huang H, McElfresh TA, Prosdocimo DA and Stanley WC: Impact of anaerobic glycolysis and oxidative substrate selection on contractile function and mechanical efficiency during moderate severity ischemia. Am J Physiol Heart Circ Physiol 295: H939-H945, 2008.

18. von Kleist-Retzow JC, Hornig-Do HT, Schauen M, Eckertz S, Dinh TA, Stassen F, Lottmann N, Bust M, Galunska B and Wielckens K: Impaired mitochondrial $\mathrm{Ca}^{2+}$ homeostasis in respiratory chain-deficient cells but efficient compensation of energetic disadvantage by enhanced anaerobic glycolysis due to low ATP steady state levels. Exp Cell Res 313: 3076-3089, 2007. 